\title{
The clinical significance of lymph node size in colon cancer
}

\author{
Bruno Märkl ${ }^{1}$, Janine Rößle ${ }^{1}$, Hans M Arnholdt ${ }^{1}$, Tina Schaller ${ }^{1}$, Ines Krammer ${ }^{1}$, \\ Claudio Cacchi ${ }^{1}$, Hendrik Jähnig ${ }^{1}$, Gerhard Schenkirsch ${ }^{2}$, Hanno Spatz ${ }^{3}$ and \\ Matthias Anthuber ${ }^{3}$
}

${ }^{1}$ Institute of Pathology, Klinikum Augsburg, Augsburg, Germany; ${ }^{2}$ Clinical and Population-Based Cancer Registry, Augsburg, Germany and ${ }^{3}$ Department of Visceral-and Transplantation-Surgery, Klinikum Augsburg, Augsburg, Germany

\begin{abstract}
To date, the clinical value of lymph node size in colon cancer has been investigated only in a few studies. Only in radiological diagnosis is lymph node size routinely recognized, and nodes $\geq 10 \mathrm{~mm}$ in diameter are considered pathologic. However, the few studies regarding this topic suggest that lymph node size is not a reliable indicator of metastatic disease. Moreover, we hypothesized that increasing lymph node size is associated with favorable outcome. By performing a morphometric study, we investigated the clinical significance of lymph node size in colon cancer in terms of metastatic disease and prognosis. A cohort of 237 cases with excellent lymph node harvest (mean lymph node count: $33 \pm 17$ ) was used. The size distribution in node-positive and -negative cases was almost identical. In all, 151 out of the 305 metastases detected (49.5\%) were found in lymph nodes with diameters $\leq 5 \mathrm{~mm}$. Only $25 \%$ of lymph nodes $>10 \mathrm{~mm}$ showed metastases. Minute lymph nodes $\leq 1 \mathrm{~mm}$ were involved only very rarely (2 of 81 cases). In $67 \%$ of the cases, the largest positive lymph node was $<10 \mathrm{~mm}$. The prognostic relevance of lymph node size was investigated in a subset of 115 stage I/II cases. The occurrence of $\geq 7$ lymph nodes that were $>5 \mathrm{~mm}$ in diameter was significantly associated with better overall survival. Our data show that lymph node size is not a suitable factor for preoperative lymph node staging. Minute lymph nodes have virtually no role in correct histopathological lymph node staging. Finally, large lymph nodes in stage $\mathrm{I} / \mathrm{II}$ disease might indicate a favorable outcome.

Modern Pathology (2012) 25, 1413-1422; doi:10.1038/modpathol.2012.92; published online 8 June 2012
\end{abstract}

Keywords: colon cancer; fat clearance; lymph node; methylene blue; morphometry

Histological lymph node staging is of crucial importance for prognosis estimation and postoperative therapy stratification. It is still the strongest prognostic parameter in curative cases. ${ }^{1}$ In nodepositive colon cancer, adjuvant chemotherapy is generally recommended. In contrast, the benefit in stage II cancers is minimal, and the decision of whether to use an adjuvant therapy depends on additional risk factors. ${ }^{2}$ Insufficient lymph node harvest is one of these factors. ${ }^{3}$ However, the number of lymph nodes needed for sufficient lymph node staging is still a matter of debate. The different recommendations range from 9 to $>30$ lymph nodes. $^{4-7}$ The Union for International Cancer Control demands the investigation of at least 12 lymph

Correspondence: Dr B Märkl, MD, PD, Institute of Pathology, Klinikum Augsburg, Stenglinstrasse 2, 86156 Augsburg, Germany. E-mail: Bruno.Maerkl@klinikum-augsburg.de

Received 16 November 2011; revised 9 April 2012; accepted 12 April 2012; published online 8 June 2012 nodes. ${ }^{8}$ Nevertheless, many studies have found that this standard is not kept in daily routine..$^{4,6,9,10}$ Fat clearance methods are well known to improve lymph node harvest in colorectal cancer. ${ }^{11,12}$ Unfortunately, these protocols are time consuming and expensive. Recently, we introduced methylene blueassisted lymph node dissection as a cheap and highly effective method to guarantee optimal lymph node staging in gastrointestinal cancers, with lymph node numbers by far exceeding the general recommendations. ${ }^{13-16}$ This improvement is mainly the result of better visibility of small lymph nodes. Therefore, the question arises as to whether the detection of such small lymph nodes is clinically significant. The metastatic involvement of lymph nodes as small as $1 \mathrm{~mm}$ is described in the literature. ${ }^{17}$ However, the clinical significance of such findings is unclear. Radiologists screen their images for the occurrence of large lymph nodes $(\geq 10 \mathrm{~mm})$ in order to detect metastatic diseases. ${ }^{18}$ Our impression, however, is that larger lymph nodes 
are often not involved in metastatic disease but show lymphofollicular hyperplasia. ${ }^{19-23}$ This might explain the relatively disappointing results of diagnosis based on radiological lymph node status assignment, which is mainly dependent upon lymph node size. ${ }^{24}$ Moreover, one could hypothesize that lymphatic hyperplasia in colon cancer could be a prognostic marker indicating an especially effective immunological response against the cancer. ${ }^{25-28}$ As large lymph nodes are easier to find, this could be an alternative explanation for the phenomenon that high lymph node counts are associated with a favorable outcome in stage II and even in stage III colon cancer. ${ }^{29}$ Until now, insufficient surgical techniques and, even more likely, stage migration are thought to explain this well-investigated effect. ${ }^{4,30,31}$ Following this hypothesis, missed lymph node metastases in cases with insufficient or moderately sufficient lymph node harvest would lead to a poorer outcome in alleged node-negative (stage II) cases.

On the basis of these considerations, we performed a morphometric study on a collection of colon cancer cases with optimized lymph node dissection by the methylene blue technique or fat clearance. The main goals of this study were to elucidate the roles of minute and enlarged lymph nodes. Moreover, we tested the hypothesis that factors displaying lymph node enlargement are associated with a favorable outcome. As a result of the already mentioned disappointing results of radiological lymph node metastases detection based on lymph node size, border irregularity and heterogeneity of signal intensity has been used as alternative criteria to detect metastases by magnetic resonance imaging. ${ }^{32}$ In order to evaluate whether the criterion is reproducible histologically, we performed an additional investigation on a subset of 28 nodal-positive cases.

\section{Patients and methods}

\section{Patients}

In all, 237 patients were enrolled between May 2007 and August 2010. Inclusion criteria were surgical resection of colon cancers with curative intent, tumor-free resection margins and the application of either the methylene blue technique or fat clearance. Exclusion criteria were rectal cancer, emergency non-elective resection, primary palliative resection and inflammatory bowel disease. In total, 49 cases were part of a foregoing study. ${ }^{14}$ The other cases were collected prospectively afterward. Follow-up data have been provided by the clinical and population-based cancer registry Augsburg. Follow-up data were missing in 45 cases.

For the analysis of the hypothesized prognostic effect of enlarged lymph nodes, a subgroup of nodenegative cases was built. After exclusion of cases with missing follow-up or follow-up shorter than 2 months, this group included 115 cases.

\section{Lymph Node Dissection}

The techniques have been described previously and will be mentioned here only briefly. ${ }^{14}$

\section{Methylene blue-assisted lymph node dissection}

After receiving the specimens in a fresh or shortly fixed $(<3 \mathrm{~h})$ state, the main artery was identified and $15-20 \mathrm{ml}$ of methylene blue solution (diluted with saline 1:3) was gently injected. Then the specimens were fixed overnight in $10 \%$ formalin. On the next day after cutting out a routine sample of the tumor and the resection margins, the mesenteric adipose tissue was separated from the bowel wall and sliced into thin sections. Lymph nodes of all sizes were highlighted by methylene blue staining and, therefore, were easy to detect.

\section{Fat clearance}

The specimens were treated in the conventional manner after fixing overnight. Lymph nodes were dissected manually and the remaining adipose tissue then underwent a fat clearing procedure. The protocol included an isopropanol treatment for 3 days with increasing concentrations and a final xylene step for one day. After this process, a secondary lymph node dissection was performed. The primary and secondary lymph nodes were analyzed in this study.

\section{Morphometric Analysis}

Morphometric analysis was performed on hematoxylin and eosin (H\&E)-stained slides using routine microscopes and digital cameras with calibrated software systems (ProgRes C10 and C3, Jenoptik, Jena, Germany) for measurements of diameters up to $8 \mathrm{~mm}$. Larger diameters were measured by marking the areas of interest under the microscope and measuring with a ruler. Both metastases and micrometastases $(0.2-2 \mathrm{~mm})$ were counted as nodal positive. The lymph nodes were categorized according to their maximum diameters into 1 of 11 categories (1-10 with $1 \mathrm{~mm}$ steps and $>10 \mathrm{~mm}$ ). For each case, a cumulative lymph node diameter (cLND) was calculated by adding all lymph node diameters. An arbitrarily chosen value of $12 \mathrm{~mm}$ was used for the last category $(>10 \mathrm{~mm})$. The parameter LN5 represents lymph nodes with diameters $>5 \mathrm{~mm}$.

\section{Lymph Node Border Irregularity}

The H\&E-stained slides of the lymph nodes of 28 nodal-positive cases were screened macroscopically by one pathologist (BM). Border irregularity was 
defined as macroscopically recognizable deviation from ideal border contour. Irregular lymph nodes were categorized as metastasized or negative.

\section{Statistics}

The unpaired T-test was used to compare normal distribution parameters of two groups. If the normal distribution was not given, then a Mann-Whitney rank sum test was performed instead. For comparison of $>2$ variables one-way RM ANOVA test was used. Dichotomous data were compared using the $\chi^{2}$ test or Fisher's exact test. Mean values are given \pm 1 s.d. Optimal cutoff values (with high sensitivity for progression-free survival) were determined using receiver operating characteristic analysis Figure $2 b$. Kaplan-Meier analysis and log-rank test was performed to compare the overall survival between different groups. Forward and backward stepwise regression was performed to estimate the influence of different established prognostic and lymph noderelated factors. A $P$-value of $<0.05$ was considered significant. All calculations were performed using the Sigma Plot 11.0 software package (Systat, Richmond, VA, USA).

\section{Results}

\section{Size Distribution of Lymph Nodes-All Stages}

The clinico-pathological data are summarized in Table 1. The size distribution of all 7877 lymph nodes that were analyzed is shown in Figure 1a. The majority of lymph nodes measured between $>1$ and $\leq 5 \mathrm{~mm}$, whereas larger lymph nodes were found at a considerably lower frequency. The comparison of node-negative with node-positive cases reveals an almost identical size distribution in both subgroups
(Figure 1b). Only the proportion of $4 \mathrm{~mm}$-sized lymph nodes is significantly larger $(P=0.041)$ in the node-positive group. The number of large lymph nodes, in particular, seems not to be influenced by metastatic spread. Figure 1c shows the size distribution of the 305 metastasized lymph nodes and the proportions of the different sizes based on the number of all lymph nodes that were found. Metastases occurred in lymph nodes of all sizes. However, metastases in lymph nodes $<1 \mathrm{~mm}$ were rare. Only 2 out of a total of 413 lymph nodes of this minute size were positive. The proportion of positive lymph nodes increased with the diameter. In all, 151 out of the 305 detected metastases $(49.5 \%)$ were found in lymph nodes with diameters $\leq 5 \mathrm{~mm}$. The highest proportion of positive lymph nodes was found in the $>10 \mathrm{~mm}$ category with $27 \%$ positive lymph nodes (Figure 1c).

The sizes of the largest involved lymph nodes of the 81 node-positive cases are shown in Figure 1d. Interestingly, the largest positive lymph nodes in two cases were only 2 and $3 \mathrm{~mm}$ in diameter. In 23 of 81 cases $(28 \%)$, the largest lymph node was $>10 \mathrm{~mm}$. The diameters of the largest positive lymph nodes that defined the final nodal status are also given in Figure 1d. In total, 43 (53\%) of these lymph nodes were $\leq 5 \mathrm{~mm}$ (Figure $1 \mathrm{~d}$ ).

\section{Prognostic Effect of Lymph Node Size in Node- Negative Cancer}

The clinico-pathological characteristics of node negative cases are given in Table 2. Total lymph node number (tLN), number of lymph nodes $>5 \mathrm{~mm}$ (LN5) and cLND have been investigated concerning their association with tumor progression. The linear regression analysis revealed a strong dependency between tLN and cLND (Rsqr $=0.815)$. In contrast,

Table 1 Clinico-pathological characteristics

\begin{tabular}{|c|c|c|c|c|}
\hline & All cases $(\mathrm{n}=237)$ & Node negative $(\mathrm{n}=115)$ & LN5-low $(\mathrm{n}=86)$ & LN5-high $(\mathrm{n}=29)$ \\
\hline Mean Age \pm s.d. & $69 \pm 12$ & $71 \pm 13$ & $70 \pm 11$ & $66 \pm 12$ \\
\hline younger than 50 years & 17 & 6 & 3 & 3 \\
\hline Female-male ratio & $1: 1.37$ & $1: 1.61$ & $1: 1.61$ & $1: 1.63$ \\
\hline Hemicolon right & 90 & 39 & 17 & 22 \\
\hline Colon transversum & 6 & 1 & 0 & 1 \\
\hline Hemicolon left & 23 & 13 & 10 & 3 \\
\hline Sigmoid colon & 117 & 61 & 58 & 3 \\
\hline Complete colon & 1 & 1 & 1 & 0 \\
\hline pT1 & 26 & 18 & 16 & 2 \\
\hline pT2 & 44 & 26 & 18 & 8 \\
\hline pT3 & 145 & 66 & 48 & 18 \\
\hline pT4 & 22 & 17 & 16 & 1 \\
\hline Mean LN count \pm s.d. & $33 \pm 17$ & $37 \pm 18$ & $29 \pm 13$ & $46 \pm 23$ \\
\hline Node positive & 81 & - & - & - \\
\hline Low grade & 188 & 98 & 73 & 25 \\
\hline High grade & 49 & 17 & 13 & 4 \\
\hline Lymphatic invasion & 25 & 12 & 11 & 1 \\
\hline Venous invasion & 29 & 9 & 7 & 2 \\
\hline Methylene blue technique & 212 & 99 & 74 & 25 \\
\hline Fat clearance & 25 & 16 & 12 & 4 \\
\hline
\end{tabular}



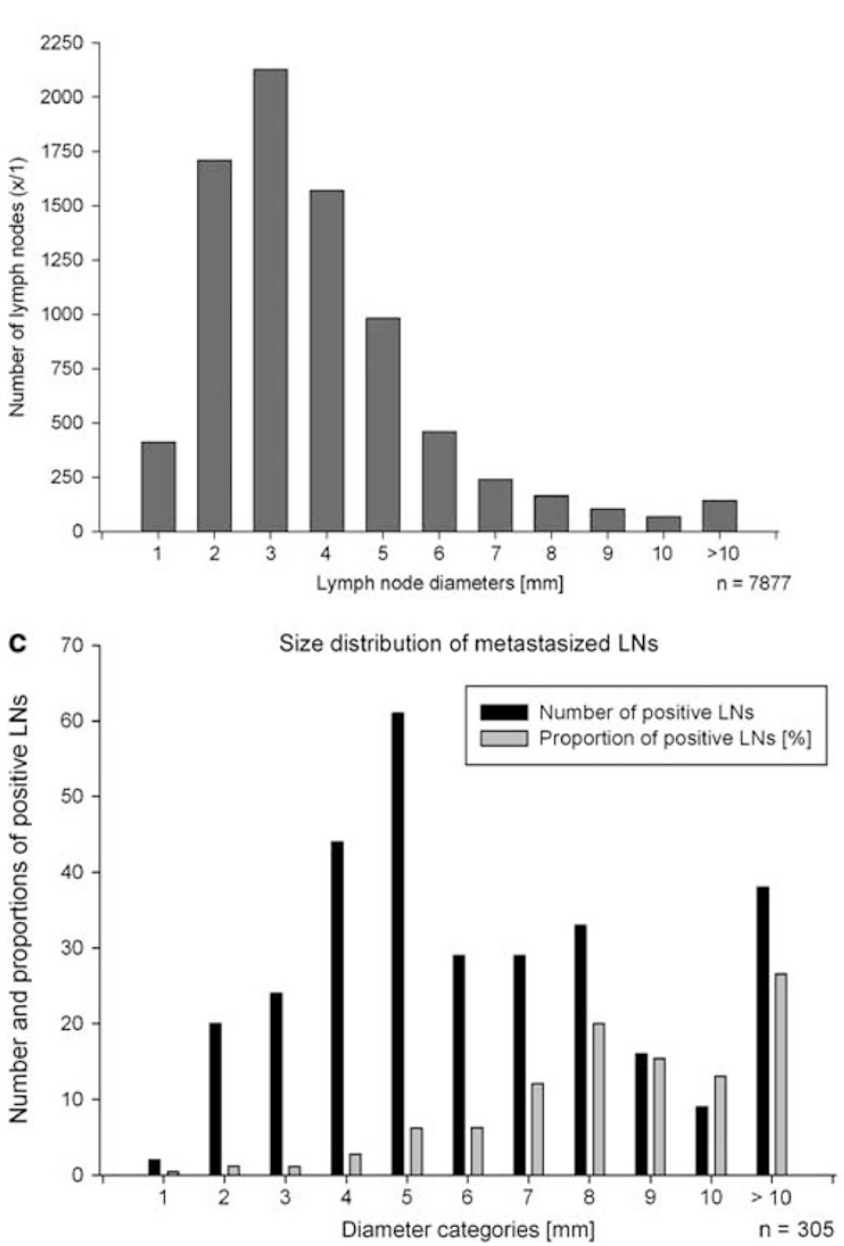

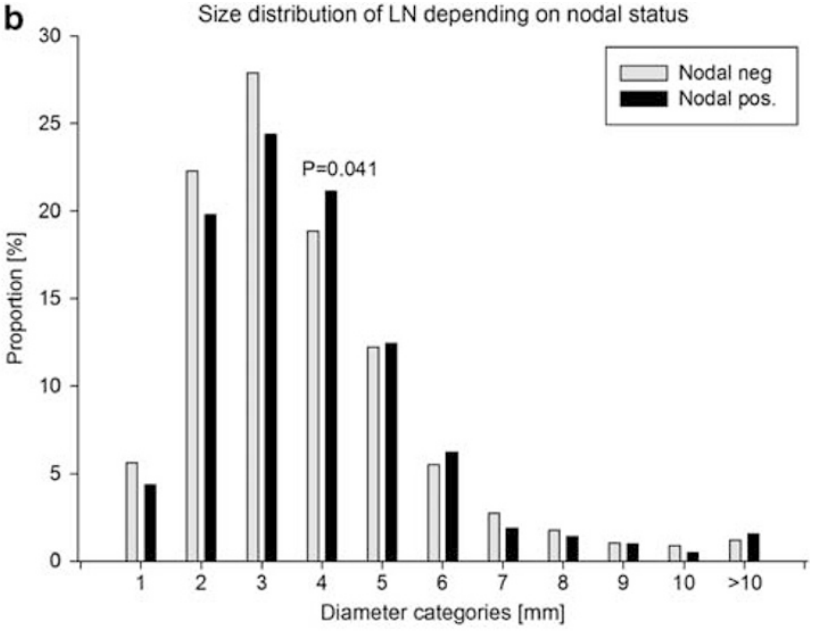

d

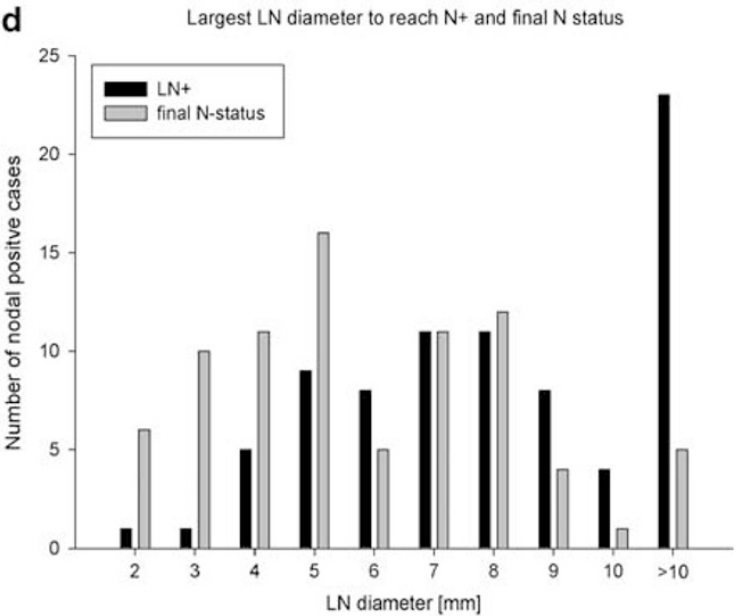

Figure 1 (a) The graph shows the distribution of the lymph node sizes of all lymph nodes. (b) The size distribution depending of the nodal status is shown. Note: except the category of lymph nodes $4 \mathrm{~mm}$ in diameter there are no significant differences. (c) The size distribution of metastasized lymph nodes in absolute numbers and proportions based on the total number of detected lymph nodes in each category is given. (d) This graph shows a distribution of the largest metastasized lymph node each. The gray bars show the largest lymph node for the final $\mathrm{N}$ status $(\mathrm{eg}, 2 \mathrm{~b})$.

Table 2 Clinico-pathological characteristics—node-negative cases

\begin{tabular}{|c|c|c|c|c|c|c|}
\hline & $\begin{array}{c}\text { Mean } L N \\
\text { count }\end{array}$ & $\mathrm{P}$-value & $\begin{array}{l}\text { Number of } \\
L N>5 \mathrm{~mm}\end{array}$ & $\mathrm{P}$-value & $\begin{array}{l}\text { Cummulative } \\
\text { LN diameter }\end{array}$ & $\mathrm{P}$-value \\
\hline Male $(n=71)$ & $33.2 \pm 17.2$ & & $4.6 \pm 5.0$ & & $125.6 \pm 74.2$ & \\
\hline Female $(n=44)$ & $33.0 \pm 18.5$ & NS & $4.2 \pm 3.9$ & NS & $124.5 \pm 76.3$ & NS \\
\hline Age $<=50(n=6)$ & $55.7 \pm 35.7$ & & $9.8 \pm 10.0$ & & $226.2 \pm 170.8$ & \\
\hline Age $>50(n=109)$ & $31.8 \pm 15.5$ & 0.09 & $4.1 \pm 4.0$ & 0.14 & $119.6 \pm 63.1$ & 0.093 \\
\hline pT1 $(n=18)$ & $27.6 \pm 13.8$ & & $2.2 \pm 3.4$ & & $92.4 \pm 64.0$ & \\
\hline pT2 $(n=26)$ & $42.1 \pm 21.5$ & & $4.8 \pm 4.2$ & & $150.9 \pm 83.9$ & \\
\hline pT3 $(n=66)$ & $30.7 \pm 16.1$ & & $4.8 \pm 4.8$ & & $122.4 \pm 71.6$ & \\
\hline $\mathrm{pT} 4(n=5)$ & $37.4 \pm 11.5$ & $<0.001$ & $5.8 \pm 7.2$ & NS & $146.2 \pm 73.5$ & $<0.001$ \\
\hline Low grade $(n=95)$ & $32.8 \pm 18.3$ & & $4.3 \pm 4.3$ & & $123.5 \pm 76.8$ & \\
\hline High grade $(n=20)$ & $34.8 \pm 13.7$ & NS & $5.3 \pm 6.0$ & NS & $133.6 \pm 66.0$ & NS \\
\hline Right colon $(n=75)$ & $37.2 \pm 18.3$ & & $7.6 \pm 4.7$ & & $160.2 \pm 73.9$ & \\
\hline Left colon $(n=40)$ & $30.9 \pm 16.8$ & 0.029 & $2.8 \pm 3.7$ & $<0.001$ & $106.5 \pm 68.5$ & $<0.001$ \\
\hline
\end{tabular}

the dependency between LN5 and tLN was considerably weaker $(\mathrm{Rsqr}=0.325)$ (Figure 2a). Comparing CLND and LN5 in node-negative cases with and without development of distant metastases revealed marginally significant lower mean values in progressive disease (cLND: $130 \pm 80$ vs $95 \pm 43$, 
a

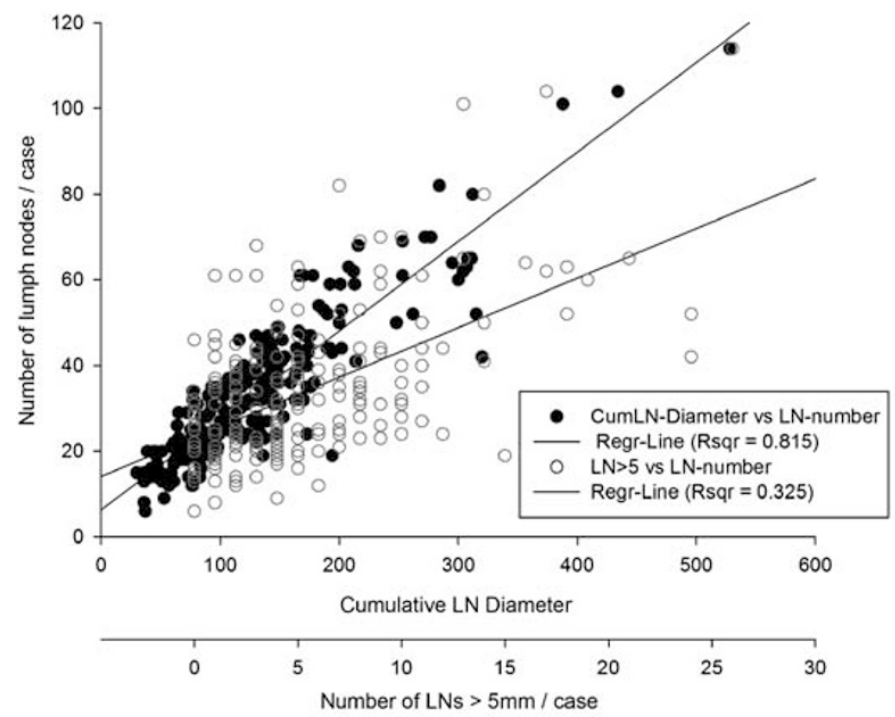

C

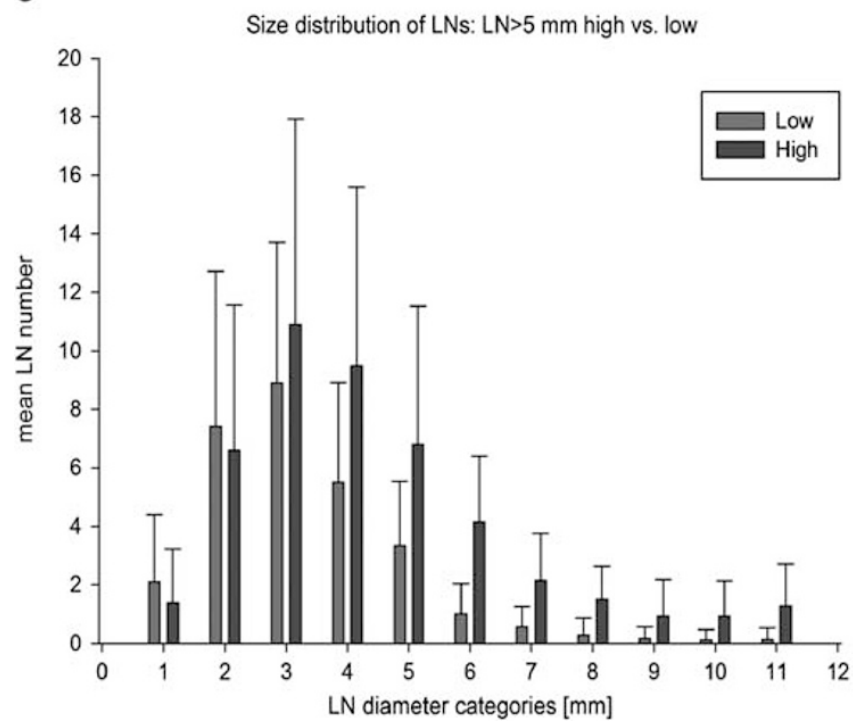

b

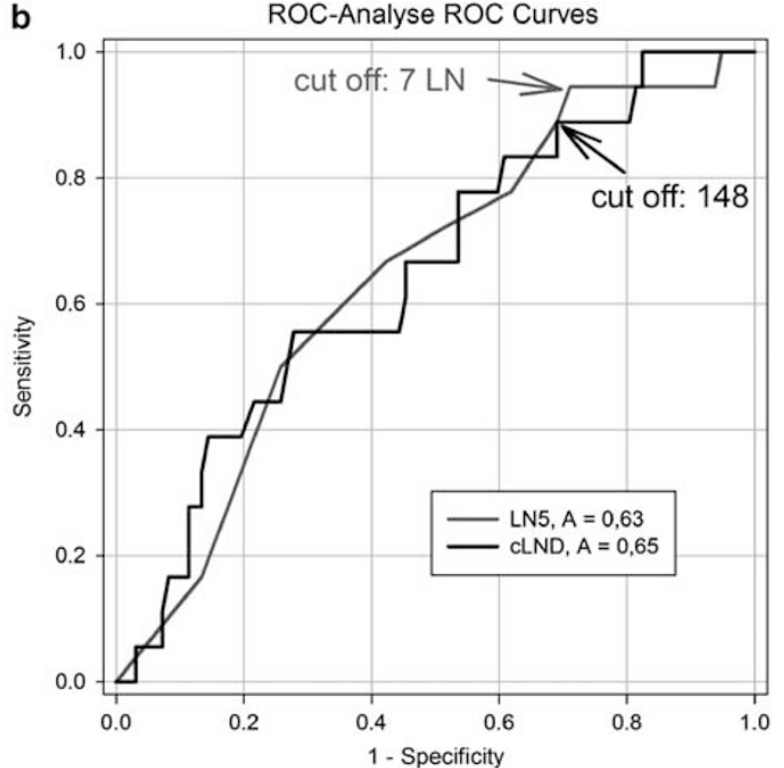

d

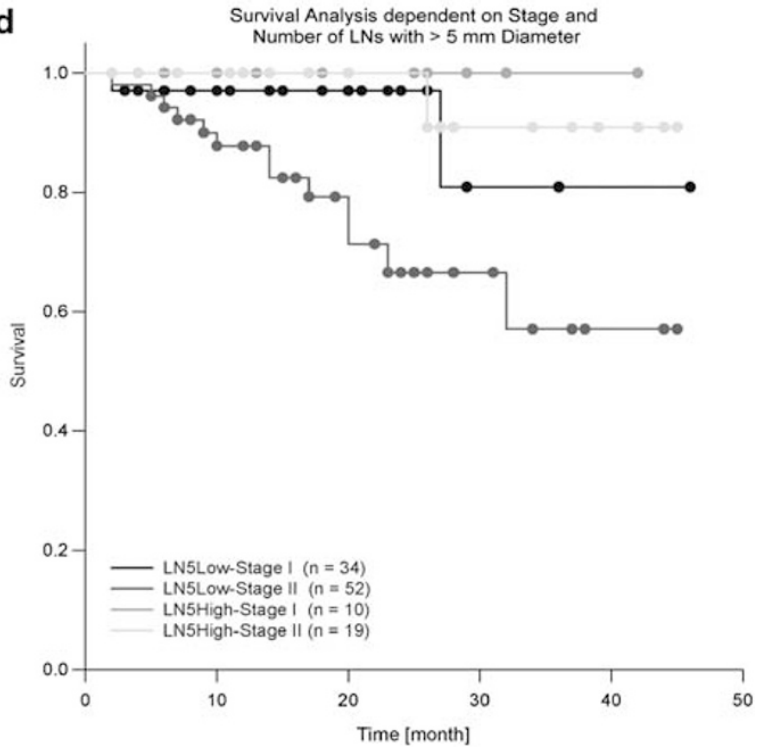

Figure 2 (a) The figure shows the regression of tLN vs LN5 and cLND, respectively. tLN and cLND (black dots) show a strong association. The association between ILN and LN5 is considerable weaker. (b) LN5 = lymph nodes > $5 \mathrm{~mm}$; cLND, cumulative lymph node diameter. Receiver operating characteristic curve to identify an optimal cut off for the parameters LN5 and cLND. The goal was to reach a high sensitivity for progression-free survival. (c) Size distribution of the LN5-high (cases with $\geq 7$ lymph nodes with diameters $>5$ mm) and LN5-low (cases with $<7$ lymph nodes with diameters $>5 \mathrm{~mm}$ ) group-all cases belong to stage I or II cancers. LN5-high cases show a significant shift toward larger lymph nodes. (d) Survival analysis of LN5-high vs -low divided into stage I and stage II cases. LN5-high cases show a better outcome in comparison with LN5-low cases. The difference between stage II cases is significant $(P=0.033)$.

$P=0.052$; LN5: $5 \pm 5$ vs $3 \pm 3 P=0.052$ ). On the other hand, no significant differences were found in nodepositive cases.

The receiver operating characteristic analysis performed to establish optimal cutoff values for the prediction of distant metastases in stage I and II cases showed similar areas under the curve for LN5 and cLND with 0.63 and 0.65 , respectively. The optimal cutoffs for predicting progressive survival for LN5 and cLND are 7 and 148, respectively. As a result of the weaker dependency on tLN, further investigations were performed with the parameter
LN5. Using the cutoff value of $\geq 7$ LN5, there was a clear trend toward fewer cases with progressive disease in the group with a high number of LN5 ( 1 out of 28 vs 17 out of $69 ; P=0.072$ ). The outcome of the LN5-high group was significantly better compared with the LN5-low group $(P=0.024)$. This is particularly the case when the cohorts are subdivided in pT1/2 and pT3/4 cases $(P=0.033$ for the comparison of pT3/4 subgroups) Figure 2d. A multivariable regression analysis included the parameters T-stage, grading, age, location, LN5 and tLN. The occurrence of distant metastases can be 
predicted from a linear combination of the T-stage $(P<0.001)$ and LN5 $(P<0.020)$ factors. A second analysis including the variables LN5, T-stage, grading, lymphatic and vascular invasion revealed LN5 $(P=0.04)$, T-stage $(P=0.004)$ and vascular invasion $(P=0.002)$ as independent predictors for the occurrence of distant metastases.

The size distribution analysis shows a general shift toward larger lymph nodes in the LN5-high group (Figure 2c). The subgroup characteristics are also given in Table 1. The main differences between the subgroups occurred with regard to T-stage, mean tLN and tumor localization. The majority of LN5high cases showed pT2 and pT3 stages, whereas pT1 and pT4 stages were considerably more frequent in the LN5-low group. The most striking finding, however, is the different side distribution. LN5low shows a dominance of left colon cancers. In contrast, 23 out of 29 cancers (79\%) in the LN5-high group occurred in the right colon. To rule out the possibility that the survival advantage of the LN5high group is a function of the location, a KaplanMeier analysis was performed comparing left and right colon cancer, which revealed no difference concerning the overall survival $(P=0.478)$ between left- and right-sided colon cancer. Information regarding immunohistochemical MLH1 expression in patients older than 50 years and microsatellite
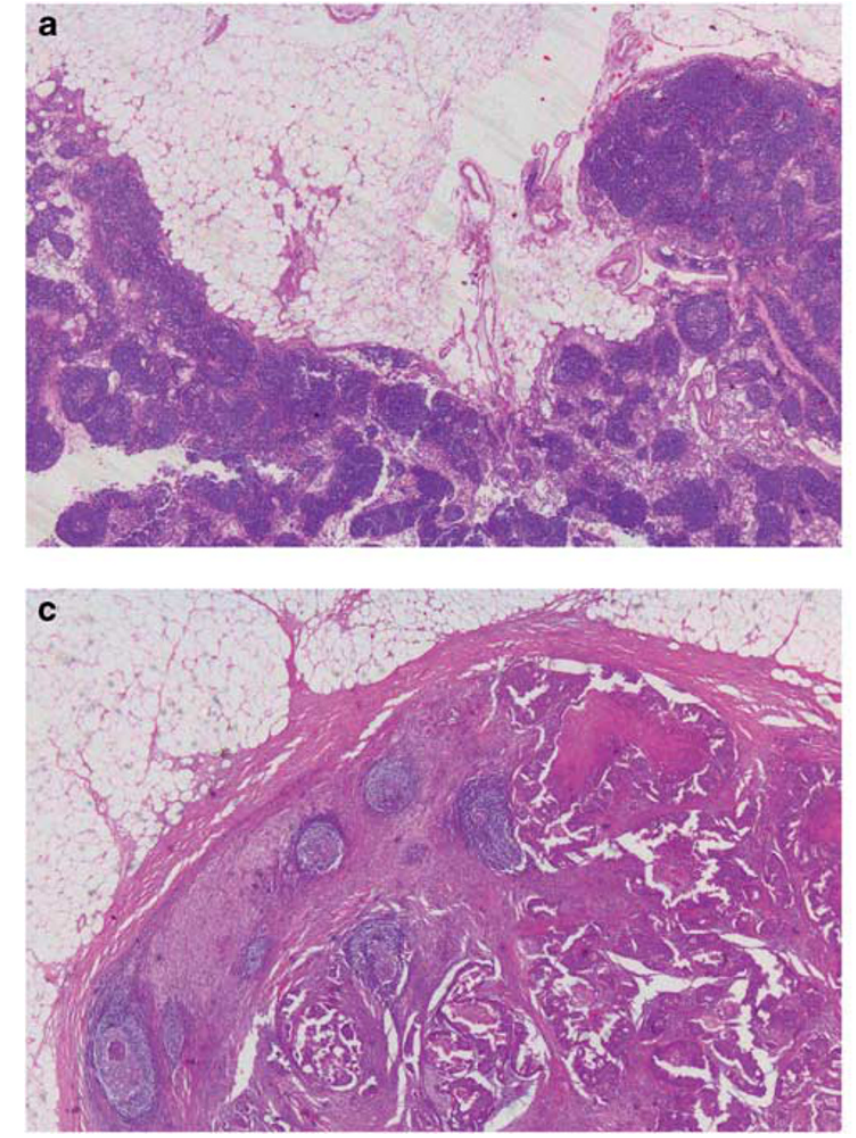

status in younger patients were available in 25 out of 29 cases in the LN5-high group. Seven cases showed loss of MLH1 expression or microsatellite instability. These cases, however, did not significantly differ concerning the number of lymph nodes with diameters $>5 \mathrm{~mm}$ within this group. Compared with the LN5-low group, the LN5-high group showed a highly significantly higher mean LN count (45 \pm 23 vs $23 \pm 13 ; P<0.001$ ), the minimal LN count was 24 in 3 cases.

\section{Lymph Node Border Irregularity}

Border irregularity was found in 22 of 116 (19\%) metastasized lymph nodes and in 5 of $1170(0.4 \%)$ negative lymph nodes. At least one irregular positive lymph node was found in 13 of 28 (46\%) cases. The irregular negative lymph nodes occurred in three cases (11\%). Irregularity occurred only in lymph nodes $>5 \mathrm{~mm}$ (Figure 3 ).

\section{Discussion}

Correct lymph node staging is of paramount importance for prognosis estimation and therapy stratification in colon cancer. ${ }^{1}$ A large number of studies address the topics of optimal lymph node

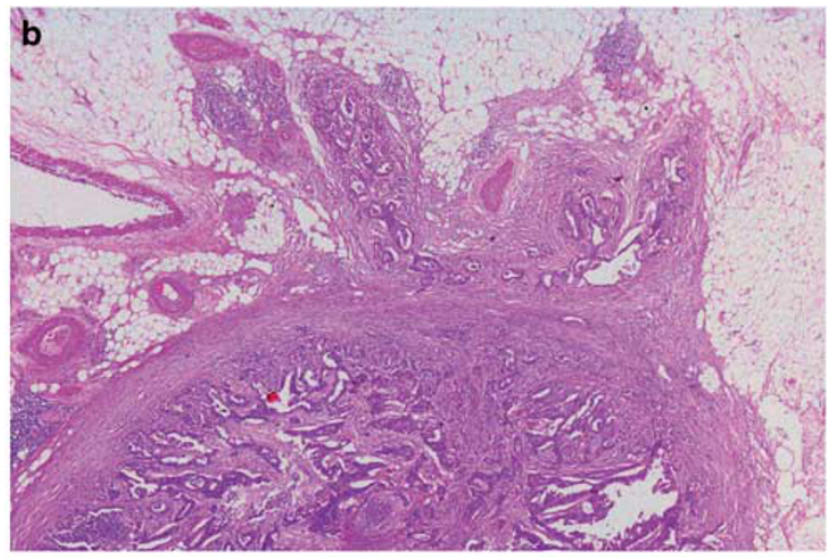

Figure 3 (a) Colonic lymph node without metastasis with considerably irregular border (H\&E 16). (b) Irregular contour of a metastasized colonic lymph node (H\&E 16). (c) Preserved regular contour of a metastasized colonic lymph node (H\&E 16). 
counts, dissecting techniques, factors influencing the lymph node harvest, lymph node ratio and the association between lymph node numbers and outcome. $^{33}$ The size of lymph nodes, however, has received attention in only a few studies. ${ }^{20-23}$ Using advanced lymph node dissection techniques, we collected a relatively large number of colon cancer cases with optimal lymph node staging starting in $2007 .{ }^{14}$ As the study was conducted at a single center and a standardized dissection technique was used, we were able to reduce confounding factors that might hamper large database studies. In this context the question may rise whether the results of this study are transferrable to routine circumstances where usually far fewer lymph nodes were harvested. However, the role of different lymph node sizes in colorectal cancer is only to investigate using a cohort that represents the whole spectrum of lymph nodes. Regarding the clinical meaning of enlarged lymph nodes, we are convinced that the dissection technique has a minor role because they hardly will be missed during routine dissection.

The first part of our study dealt with the questions: how are the sizes of lymph nodes distributed, which lymph nodes are affected by metastases, do the size distributions between nodepositive and -negative cases differ, and what are the roles of minute lymph nodes $(\leq 1 \mathrm{~mm})$ and large lymph nodes? The second part investigated a hypothesized association between lymph node size and outcome.

Our study has been restricted to colon cancer because preoperative radiation therapy, which is often applied in rectal cancer, influences lymph node staging in several aspects. ${ }^{34}$

Most of the lymph nodes detected were found in the groups between $>1$ and $\leq 5 \mathrm{~mm}$. Interestingly, the size distribution differs only slightly between node-positive and -negative cases. Only the proportion of lymph nodes between 3 and $4 \mathrm{~mm}$ is significantly larger in node-metastasized cancer. Cserni et $a l^{20}$ as well as Mönig et al found significant larger mean lymph node diameters in node-positive colorectal cancer. ${ }^{22}$ However, the difference was very small $(4.3$ vs $4.7 \mathrm{~mm} ; P<0.01$ and 3.9 vs $5.9 \mathrm{~mm} ; P<0.0001)$ and, therefore, probably not of clinical relevance. In our study, metastases occurred in lymph nodes of all sizes. About $50 \%$ of the metastases were found in lymph nodes $<6 \mathrm{~mm}$. This stands in concordance with the data of Cserni et $a .^{20}$ The likelihood of detecting a metastasis clearly increases with increasing lymph node size. However, even in the group $>10 \mathrm{~mm}$ the rate of positivity is only $28 \%$. In other words, $72 \%$ of very large lymph nodes are negative, and the vast majority of positive nodes are $<10 \mathrm{~mm}$ in diameter $(73 \%)$. As radiological lymph node staging is mainly based on the detection of large lymph nodes $>10 \mathrm{~mm}$, this finding is of clinical importance. It could explain why the results of radiological imaging in this field are somewhat disappointing. ${ }^{24}$
When comparing radiological and histopathological measurements, one has to keep in mind that lymph nodes are prone to shrink during the histological processing. Based on our observations, we estimate this effect to be in the range of about $10 \%$. Taking that into account, one can assume that lymph nodes measuring 8-10 mm histologically are already beyond the $10 \mathrm{~mm}$ threshold, which would alter the sensitivity. The specificity might still be low. Our preliminary data concerning the association between irregularities of lymph node's border and the occurrence of metastases are also not convincing. Using this criterion more than the half of cases would have been understaged, whereas negative lymph nodes with border irregularities were detected in $11 \%$.

Although the detection of each additional positive lymph node has an impact on the patient's prognosis and, therefore, is relevant, the lymph node discovered first is most important because it changes the therapy. The clinical significance of a $3 \mathrm{~mm}$ positive lymph node changes in light of a $12 \mathrm{~mm}$ positive lymph node that exists simultaneously. Therefore, we investigated the size of the largest lymph node in each node-positive case. This analysis shows that most of the largest lymph nodes affected are $>5 \mathrm{~mm}$ in diameter. Therefore, they should be easy to detect during pathological dissection. Nevertheless, a small proportion of cases (about $2.5 \%$ ) carry metastases only in very small lymph nodes (Figure 1d), which can be easily missed during routine dissection and are prone to understaging. The detection of small lymph nodes is especially important for exact substaging. In all, $53 \%$ of the lymph nodes defining the final nodal status according to the current TNM classification were $<6 \mathrm{~mm}$. Lymph nodes $<1 \mathrm{~mm}$, however, seem to have no role in this context. Only 2 out of 305 affected lymph nodes were that small. Similar findings were reported by Brown et al. ${ }^{17}$

Several studies showed that the number of lymph nodes investigated is of remarkable prognostic relevance mainly in stage II but also in stage III colon cancer. There are three potential factors that could influence lymph node harvest and prognosis: the surgeon, the pathologist and the patient. An insufficient surgical technique can lead to an incomplete lymph node resection. The clinical significance of an optimal surgical technique has been shown by Hohenberger et al. ${ }^{35}$ Unquestionably, the pathologist significantly influences the number of lymph nodes that are harvested, as long as he/she is provided with an adequate specimen. Diligence and the use of advanced methods like fat clearance or methylene blue technique have an important role. ${ }^{12,14}$ By investigating too few lymph nodes, a pathologist runs the risk of understaging. We detected the phenomenon of understaging even in primarily sufficient investigated cases using the fat clearance technique after initial conventional dissection. ${ }^{14}$ The question, however, is how often does 
that occur? And is this frequency in a range that could explain sufficiently the much better prognosis of patients with a high number of investigated lymph nodes? We suppose that only small lymph node metastases $(<5 \mathrm{~mm})$ will escape detection. Our data show that this risk occurs in about $9 \%$ of metastasized cases and in $3 \%$ of the entire collection.

On the basis of our experiences, we estimate the rate of understaging in daily routine to be between 2 and $5 \%$. In our opinion, this rate is too small to be the major reason for the large outcome difference between high and low lymph node counts. Swanson et $a l^{30}$ found a $69 \%$ 5-year survival rate for patients with pT3No colon cancers with $<5$ examined lymph nodes. Taking into account that these patients perhaps received no adjuvant therapy, about $90 \%$ must have had occult lymph node metastases in order to explain this adverse outcome. However, this percentage seems unreasonably high. Simunovic and Baxter ${ }^{36}$ supposed in an editorial that an unknown confounder might be the true explanation for the association between lymph node harvest and outcome. We now strongly believe that the patient, or rather the patient's response to the cancer, could be this confounder. Several other authors speculated in the past that the success in harvesting lymph nodes could be triggered by the lymph node size..$^{9,25,27,37-39}$ Large lymph nodes, on the other hand, could be the expression of an enhanced immunological defense.

We investigated the hypothesis that the occurrence of large lymph nodes is associated with a better prognosis in the subgroup of node-negative cases. Initially, we supposed that the cLND parameter, which is calculated by adding up all lymph node diameters of a case, could indicate the immunological response to the cancer. However, this parameter showed a very strong association with the total number of harvested lymph nodes. The size distribution of the lymph nodes does not seem to be adequately expressed by this parameter. In contrast, the simple counting of lymph nodes $>5 \mathrm{~mm}$ in diameter was only very weakly associated with the tLN and, therefore, had a higher potential to offer new insights. Indeed, the LN5-high group showed only one case $(3.3 \%)$ of progressive disease with distant metastases, whereas 16 cases $(19 \%)$ of progressive disease occurred in the LN5-low group $(P=0.072)$. Interestingly, the single progressive case in LN5-high is a 74-year-old lady who is still alive 42 months after initial diagnosis. The Kaplan-Meier analysis revealed a significant better outcome in the LN5-high group. By comparing the clinico-pathological parameters between LN5-high and -low, we found a striking difference regarding the location of the tumors. The LN5-high group showed highly significantly more right than left colon cancers. Interestingly, we found no significant survival difference when we compared the entire groups of left with right cancers. Several authors report that the right location is associated with higher lymph node counts. ${ }^{9,25,27,37-39}$ This fact further suggests the previously supposed connection between lymph node size and harvest. The combination of rightsided colon cancer and strong immunological response raised the question of whether there is an association with microsatellite instability as was found by Søreide et al. ${ }^{38}$ We could discover a clear association between microsatellite instability and lymph node size in patients younger than 50 years. Analyzing the LN5-high group, we found seven microsatellite instable cases. However, there is still a group of at least 17 cases with no known explanation for the enhanced lymphatic response. This group might represent a certain subentity of colon cancers with favorable outcome and preferred right location. In this context, it should be emphasized that lymph node count is only prognostic in colon cancer but not in rectal cancer.

In summary, lymph node size is not a reliable marker for lymph node metastases. As minute lymph nodes have virtually no role, they should not be routinely included in the pathology report. Especially in cases of low lymph node harvest, reporting very small negative lymph nodes $(\leq 1 \mathrm{~mm})$ is more a whitewash of the report than a serious report. In our eyes, the paradigm of the 12 lymph node rule should pathologists not force to count these tiny lymph nodes otherwise arbitrariness is the consequence. Independent from the quality of the specimen 12 lymph nodes can be found as long as one is willing to embed enough fat and cut enough sections in order to detect very small nodes.

The detection of relatively small lymph nodes (1$5 \mathrm{~mm}$ ), however, is important for an exact lymph node staging. Moreover, our data suggest that the lymph node size is of prognostic relevance. The fact that a high number of examined lymph nodes are associated with a favorable outcome in colon cancer might be due an enhanced immunological response with enlargement of lymph nodes, which are then easier to detect in high numbers. As this part of our study is limited by a rather small study size and short follow-up we plan confirming the results by a larger sized study with longer follow-up within the next 2 years. Moreover, the prognostic value of lymph node size in rectal cancer as well as in nodalmetastasized cancers has to be evaluated in additional studies.

\section{Acknowledgements}

This work is part of Janine Rößle's doctoral thesis and has been supported by a grant of the ManfredStolte-Stiftung. We thank Jenny Müller for excellent technical assistance and are grateful to Barbara Kresse and Kai-Uwe Hebick for archival and data work. We are further grateful to Hallie Kretsinger for revising the manuscript as a native English speaker. 


\section{Disclosure/conflict of interest}

The authors declare no conflict of interest.

\section{References}

1 Compton CC. Colorectal carcinoma: diagnostic, prognostic, and molecular features. Mod Pathol 2003; 16:376-388.

2 Gill S, Loprinzi CL, Sargent DJ, et al. Pooled analysis of fluorouracil-based adjuvant therapy for stage ii and iii colon cancer: who benefits and by how much? J Clin Oncol 2004;22:1797-1806.

3 Earle CC, Weiser MR, Ter Veer A, et al. Effect of lymph node retrieval rates on the utilization of adjuvant chemotherapy in stage ii colon cancer. J Surg Oncol 2009;100:525-528.

4 Goldstein NS. Lymph node recoveries from 2427 pt3 colorectal resection specimens spanning 45 years: recommendations for a minimum number of recovered lymph nodes based on predictive probabilities. Am J Surg Pathol 2002;26:179-189.

5 Leibl S, Tsybrovskyy O, Denk H. How many lymph nodes are necessary to stage early and advanced adenocarcinoma of the sigmoid colon and upper rectum? Virchows Arch 2003;443:133-138.

6 Maurel J, Launoy G, Grosclaude P, et al. Lymph node harvest reporting in patients with carcinoma of the large bowel: a French population-based study. Cancer 1998;82:1482-1486.

7 Yoshimatsu K, Ishibashi $\mathrm{K}$, Umehara A, et al. How many lymph nodes should be examined in dukes' $b$ colorectal cancer? Determination on the basis of cumulative survival rate. Hepatogastroenterology 2005;52:1703-1706.

8 Sobin LH, Wittekind C. Digestive systeme tumors. In: Sobin LH, Wittekind C (eds). TNM Classification of Malignant Tumors 6th edn, Vol., John Wiley \& Sons: Hoboken, 2002, pp 72-76.

9 Johnson PM, Malatjalian D, Porter GA. Adequacy of nodal harvest in colorectal cancer: a consecutive cohort study. J Gastrointest Surg 2002;6:883-888; discussion 9-90.

10 Wright FC, Law CH, Last L, et al. Lymph node retrieval and assessment in stage ii colorectal cancer: a population-based study. Ann Surg Oncol 2003;10:903-909.

11 Basten O, Bandorski D, Bismarck C, et al. [Acetone compression. A fast, standardized method to investigate gastrointestinal lymph nodes]. Pathologe 2010;31:218-224.

12 Haboubi NY, Clark P, Kaftan SM, et al. The importance of combining xylene clearance and immunohistochemistry in the accurate staging of colorectal carcinoma. J R Soc Med 1992;85:386-388.

13 Kerwel TG, Spatz J, Anthuber M, et al. Injecting methylene blue into the inferior mesenteric artery assures an adequate lymph node harvest and eliminates pathologist variability in nodal staging for rectal cancer. Dis Colon Rectum 2009;52:935-941.

14 Märkl B, Kerwel TG, Jähnig HG, et al. Methylene blueassisted lymph node dissection in colon specimens: a prospective, randomized study. Am J Clin Pathol 2008;130:913-919.

15 Märkl B, Kerwel TG, Wagner T, et al. Methylene blue injection into the rectal artery as a simple method to improve lymph node harvest in rectal cancer. Mod Pathol 2007;20:797-801.

16 Törnroos A, Shabo I, Druvefors B, et al. Postoperative intra-arterial methylene blue injection of colorectal cancer specimens increases the number of lymph nodes recovered. Histopathology 2011;58: 408-413.

17 Brown HG, Luckasevic TM, Medich DS, et al. Efficacy of manual dissection of lymph nodes in colon cancer resections. Mod Pathol 2004;17:402-406.

18 Elmas N, Killi RM, Sever A. Colorectal carcinoma: radiological diagnosis and staging. Eur J Radiol 2002;42:206-223.

19 Bjelovic M, Kalezic V, Petrovic M, et al. Correlation of macroscopic and histological characteristics in the regional lymph nodes of patients with rectal and sigmoidal adenocarcinoma. Hepatogastroenterology 1998;45:433-438.

20 Cserni G. The influence of nodal size on the staging of colorectal carcinomas. J Clin Pathol 2002;55:386-390.

21 Herrera-Ornelas L, Justiniano J, Castillo $\mathrm{N}$, et al. Metastases in small lymph nodes from colon cancer. Arch Surg 1987;122:1253-1256.

22 Mönig SP, Baldus SE, Zirbes TK, et al. Lymph node size and metastatic infiltration in colon cancer. Ann Surg Oncol 1999;6:579-581.

23 Rodriguez-Bigas MA, Maamoun S, Weber TK, et al. Clinical significance of colorectal cancer: metastases in lymph nodes $<5 \mathrm{~mm}$ in size. Ann Surg Oncol 1996;3:124-130.

24 Puli SR, Reddy JB, Bechtold ML, et al. Accuracy of endoscopic ultrasound to diagnose nodal invasion by rectal cancers: a meta-analysis and systematic review. Ann Surg Oncol 2009;16:1255-1265.

25 Dillman RO, Aaron K, Heinemann FS, et al. Identification of 12 or more lymph nodes in resected colon cancer specimens as an indicator of quality performance. Cancer 2009.

26 Wong SL, Ji H, Hollenbeck BK, et al. Hospital lymph node examination rates and survival after resection for colon cancer. JAMA 2007;298:2149-2154.

27 Sarli L, Bader G, Iusco D, et al. Number of lymph nodes examined and prognosis of TNM stage ii colorectal cancer. Eur J Cancer 2005;41:272-279.

28 Vather R, Sammour T, Kahokehr A, et al. Lymph node evaluation and long-term survival in stage ii and stage iii colon cancer: a national study. Ann Surg Oncol 2009;16:585-593.

29 Chang GJ, Rodriguez-Bigas MA, Skibber JM, et al. Lymph node evaluation and survival after curative resection of colon cancer: systematic review. J Natl Cancer Inst 2007;99:433-441.

30 Swanson RS, Compton CC, Stewart AK, et al. The prognosis of $\mathrm{t} 3 \mathrm{n} 0$ colon cancer is dependent on the number of lymph nodes examined. Ann Surg Oncol 2003;10:65-71.

31 Daneker Jr GW, Ellis LM. Colon cancer nodal metastasis: biologic significance and therapeutic considerations. Surg Oncol Clin N Am 1996;5:173-189.

32 Shihab OC, Taylor F, Bees $\mathrm{N}$, et al. Relevance of magnetic resonance imaging-detected pelvic sidewall lymph node involvement in rectal cancer. Br J Surg 2011;98:1798-1804.

33 Schofield JB, Mounter NA, Mallett R, et al. The importance of accurate pathological assessment of lymph node involvement in colorectal cancer. Colorectal Dis 2006;8:460-470. 
34 Mekenkamp LJ, van Krieken JH, Marijnen CA, et al. Lymph node retrieval in rectal cancer is dependent on many factors-the role of the tumor, the patient, the surgeon, the radiotherapist, and the pathologist. Am J Surg Pathol 2009;33:1547-1553.

35 Hohenberger W, Weber K, Matzel K, et al. Standardized surgery for colonic cancer: complete mesocolic excision and central ligation-technical notes and outcome. Colorectal Dis 2009;11:354-364; discussion 64-5.

36 Simunovic M, Baxter NN. Lymph node counts in colon cancer surgery: lessons for users of quality indicators. JAMA 2007;298:2194-2195.
37 Hsu CW, Lin CH, Wang JH, et al. Factors that influence 12 or more harvested lymph nodes in early-stage colorectal cancer. World J Surg 2009;33:333-339.

38 Søreide K, Nedrebo BS, Søreide JA, et al. Lymph node harvest in colon cancer: influence of microsatellite instability and proximal tumor location. World J Surg 2009;33:2695-2703.

39 Stocchi L, Fazio VW, Lavery I, et al. Individual surgeon, pathologist, and other factors affecting lymph node harvest in stage ii colon carcinoma. Is a minimum of 12 examined lymph nodes sufficient? Ann Surg Oncol 2011;18:405-412. 